\title{
Article \\ Selection of Reference Genes for Normalization of Gene Expression in Thermobia domestica (Insecta: Zygentoma: Lepismatidae)
}

\author{
Yu Bai, Ya-Nan Lv, Mei Zeng, Pei-Yao Jia, Hu-Na Lu, Yi-Bo Zhu, Sheng Li, Ying-Ying Cui and Yun-Xia Luan *D \\ Guangdong Provincial Key Laboratory of Insect Development Biology and Applied Technology, \\ Institute of Insect Science and Technology, School of Life Sciences, South China Normal University, \\ Guangzhou 510631, China; yubai@m.scnu.edu.cn (Y.B.); 2019022482@m.scnu.edu.cn (Y.-N.L.); \\ zengmei@m.scnu.edu.cn (M.Z.); 2019022472@m.scnu.edu.cn (P.-Y.J.); 2020022807@m.scnu.edu.cn (H.-N.L.); \\ 2020022824@m.scnu.edu.cn (Y.-B.Z.); lisheng@scnu.edu.cn (S.L.); cuiyingying@m.scnu.edu.cn (Y.-Y.C.) \\ * Correspondence: yxluan@scnu.edu.cn
}

Citation: Bai, Y.; Lv, Y.-N.; Zeng, M.; Jia, P.-Y.; Lu, H.-N.; Zhu, Y.-B.; Li, S.; Cui, Y.-Y.; Luan, Y.-X. Selection of Reference Genes for Normalization of Gene Expression in Thermobia domestica (Insecta: Zygentoma: Lepismatidae). Genes 2021, 12, 21. https://dx.doi.org/10.3390/ genes12010021

Received: 24 November 2020 Accepted: 22 December 2020 Published: 25 December 2020

Publisher's Note: MDPI stays neutral with regard to jurisdictional claims in published maps and institutional affiliations.

Copyright: () 2020 by the authors. Licensee MDPI, Basel, Switzerland. This article is an open access article distributed under the terms and conditions of the Creative Commons Attribution (CC BY) license (https: / / creativecommons.org/ licenses/by/4.0/).

\begin{abstract}
Zygentoma occupies a key evolutionary position for understanding the evolution of insect metamorphosis but has received little attention in terms of genetic analysis. To develop functional genomic studies in this insect, we evaluated five candidate internal reference genes for quantitative RT-PCR (qPCR) studies from Thermobia domestica, a representative species of Zygentoma, including Actin 5C (Actin5C), Elongation factor-1 alpha (EF1A), Ribosome protein S26 (RPS26), Ribosome protein L32 (RPL32), and Superoxide dismutase 2 (SOD2), at different developmental stages, in various body parts, and under dsRNA microinjection and starvation stresses, using four algorithms (delta Ct, geNorm, NormFinder and BestKeeper) and a comparative algorithm (RefFinder). Specific suitable reference genes were recommended across specific experimental conditions, and the combination of RPS26 and RPL32 was appropriate for all tested samples. Employing our selected reference gene combination, we investigated the gene expression pattern of Myoglianin (Myo), a crucial generegulating insect metamorphosis, in ametabolous T. domestica, and demonstrated the efficiency of RNA interference (RNAi) in firebrat nymphs. This study provides a basis for reliable quantitative studies of genes and greatly benefits evolutionary and functional genomics studies in Zygentoma.
\end{abstract}

Keywords: Thermobia domestica; reference genes; expression stability; quantitative real-time PCR; RNA interference

\section{Introduction}

The basal insect order Zygentoma includes the so-called silverfish and firebrats. In contrast to winged insects, primitively wingless members of Zygentoma molt throughout their entire lives and do not undergo distinct morphological changes except for increases in size. As the extant ametabolous group closest to pterygote insects [1], Zygentoma occupies a key phylogenetic position for studying the evolution of insect metamorphosis. However, to date, Zygentoma has received little attention regarding genetic analysis. As a potential model organism of ametabolous insects, the firebrat Thermobia domestica is widely distributed worldwide. Firebrats are easy to maintain, breed, and synchronize in a laboratory. Rough genomic data from $T$. domestica have been reported recently [2], which facilitates genetic studies of T. domestica.

Accurate quantification of gene expression and assessment of gene function by silencing gene expression are needed for successful functional genomics. Quantitative RT-PCR (qPCR) has emerged as a powerful tool to measure gene expression. Many factors influence the accuracy and interpretation of qPCR, including the quantity and quality of the starting material, RNA extraction, cDNA synthesis, and other laboratory procedures. To limit variability, normalization of the data occurs by comparing target gene expression 
levels to those of reference genes. Additionally, reference genes are assumed to have stable expression across various biotic and abiotic stresses and treatments (e.g., tissues and developmental stages). Depending on the experiment, these assumptions may not be valid; indeed, recent research shows that a condition-specific reference gene for a given species needs to be identified for accurate measurements of gene expression. Because the reliability of qPCR depends largely on the reference gene(s) used to normalize the data, it is crucial to know the stability of the reference gene(s) under specific experimental conditions prior to qPCR. Generally, five statistical algorithms, the delta Ct method, geNorm, NormFinder, Best-Keeper, and RefFinder, are employed to determine the expression stabilities of candidate reference genes. Although the value for $r p 49$ has been used as a reference to investigate HR3, E75, and timeless (tim) gene expression in T. domestica with qPCR [3,4], there is no stable reference gene quantification system for $T$. domestica.

RNA interference (RNAi) results in a sequence-specific knockdown of gene expression at the posttranscriptional level, as introduced double-stranded RNAs (dsRNAs) cause the degradation of identical mRNAs. dsRNA can be introduced in different ways, but injection is the most common method to deliver dsRNA into organisms. Ohde et al. [5] attempted microinjection of Distal-less (Dll) dsRNA into embryos of T. domestica, which induced defects in the formation of outgrowths. The Tomioka laboratory has performed molecular analyses of the circadian clock in firebrats by injecting dsRNA of several clock genes into the abdomen of adult firebrats, which effectively abolishes rhythmic expression [3,4]. The postembryonic development period is essential for insect growth and the accumulation of reserves. To date, few RNAi studies on nymph stages in Zygentoma have been conducted.

Here, our goals were to comprehensively evaluate the optimal reference genes for precise quantification of mRNA transcripts of $T$. domestica in different developmental stages and various tissues, as well as under RNAi and starvation stresses, in nymphs of T. domestica. Normalized with the selected combination of internal reference genes, we evaluated the spatiotemporal expression and RNAi efficiency of Myo, a ligand in the TGF- $\beta$ signaling pathway [6,7]. These data lay a solid foundation for further genetic studies on T. domestica and provide directions for studying the evolution of insect metamorphosis.

\section{Materials and Methods}

\subsection{Insect Rearing and Sampling Strategy}

Firebrats were reared in plastic containers inside a climate chamber $\left(37^{\circ} \mathrm{C}, \sim 75 \%\right.$ relative humidity, and without light). They were fed commercial rat food, and cotton balls were necessary for them to lay eggs. The insects go through egg, nymph, and adult stages. Most eggs hatch in 12-13 days, and then nymphs undergo two pro-nymph stages (N1-2) lasting 3 days, six nymph stages (N3-8), each requiring 5 days, and a preadult stage (N9) for 7-9 days. After nine nymph instars, the insects molt into sexually mature adults (A1) and pass through 45 to 60 instars undergoing molting and reproductive cycles.

In view of the fact that we usually utilize firebrats after N6 for studies, we chose mixed specimens at nymph stages N6, N7, N8, and N9 and adult stages A1, A3, and A4 to evaluate potential reference genes for different developmental stages. In addition to the whole body (WB), four different body parts, including the head (H), prothorax (T1), mesothorax/metathorax (T23), and abdomen (A), were dissected from each instar. For dissections and tissue sampling, specimens were anesthetized with carbon dioxide. Specimens were immersed in 1xPBS ( $\mathrm{pH} 7.2$ ), and tissues were collected under a stereomicroscope. Approximately $20 \mathrm{Hs}, 20 \mathrm{~T} 1 \mathrm{~s}, 10 \mathrm{~T} 23 \mathrm{~s}, 5 \mathrm{As}$, and $5 \mathrm{WBs}$ at the N6 and N7 stages and $15 \mathrm{Hs}$, 15 T1s, 8 T23s, 4 As, and 4 WBs at the N8, N9, and A1 stages were collected for the qPCR assay. Similarly, approximately $10 \mathrm{Hs}, 10 \mathrm{~T} 1 \mathrm{~s}, 5 \mathrm{~T} 23 \mathrm{~s}, 3 \mathrm{As}$, and $3 \mathrm{WBs}$ at the A3 and A4 stages were collected for the qPCR assay, and each sample included three biological replicates. All samples were stored inside $1.5 \mathrm{~mL}$ RNase-free microfuge tubes at $-80{ }^{\circ} \mathrm{C}$ until use. 


\subsection{Identification of Candidate Reference Gene Homologs in T. domestica}

Based on previous studies of reference genes from other insects [8], five candidate genes, Actin 5C (Actin5C), Elongation factor-1 a (EF1A), Ribosome protein S26 (RPS26), Ribosome protein L32 (RPL32), and Superoxide dismutase 2 (SOD2), were selected for the identification of stable reference genes in T. domestica. A BLAST search against the transcriptome (our unpublished data) and genome data [2] of T. domestica was performed to find homologs of the five genes. Primers specific to each gene were designed individually (https: //www.ncbi.nlm.nih.gov/tools/primer-blast/index.cgi?LINK_LOC=BlastHome). The amplification efficiency $(E)$ and correlation coefficient values $\left(\mathrm{R}^{2}\right)$ of the primers were calculated by a 4-point standard curve obtained by serial dilutions of known concentrations of cDNA template (Table S1 and Figure S1). The size and sequence of each PCR product were further checked through 1\% agarose gel electrophoresis and DNA sequencing.

\subsection{Total RNA Extraction, $c D N A$ Synthesis, and $q P C R$}

Total RNA was isolated from the prepared samples using RNAiso plus reagents (Takara, Dalian, China) following the supplier's instructions. The quality and quantity of isolated RNA samples were checked by employing a NanoDrop2000 (Thermo Scientific, Waltham, MA, USA). RNA samples with a $260 / 280$ ratio between 1.80 and 2.0 and a $260 / 230$ ratio between 2.00 and 2.20 were used for qPCR analysis. An aliquot of $800 \mathrm{ng}$ of total RNA was used to synthesize first-strand cDNA using the PrimeScript ${ }^{\mathrm{TM}} \mathrm{RT}$ reagent Kit with gDNA Eraser (Perfect Real Time) (Takara, Dalian, China). The qPCR experiment was performed in accordance with the Minimum Information Required for Publication of Quantitative Real-time PCR Experiments (MIQE) Guidelines [9]. qPCR was performed on the Applied Biosystems ${ }^{\mathrm{TM}}$ QuantStudio ${ }^{\mathrm{TM}} 6$ Flex Real-Time PCR System with three biological replicates and three technical replicates. Each PCR contained $8 \mu \mathrm{L}$ of 1:20 diluted cDNA, $10 \mu \mathrm{L}$ of SYBR ${ }^{\circledR}$ Premix Ex Taq ${ }^{\mathrm{TM}}$ II (Tli RNaseH Plus) (Takara, Dalian, China), and $1 \mu \mathrm{L}$ each of forward and reverse gene-specific primers $(10 \mu \mathrm{M})$. A two-step program $\left(94{ }^{\circ} \mathrm{C}\right.$ for $3 \mathrm{~min} ; 40$ cycles of $94^{\circ} \mathrm{C}$ for $10 \mathrm{~s}$ and $56^{\circ} \mathrm{C}$ for $30 \mathrm{~s}$ ) was used for qPCR.

\subsection{Cloning, Synthesis, and Delivery of dsRNA}

Gene-specific paired primers (Table S2) were designed to amplify a 232 bp fragment corresponding to T. domestica Myoglianin (Tdmyo; obtained from our unpublished transcriptome and verified with the genome data [2]) and a $193 \mathrm{bp}$ fragment corresponding to Mus musculus lymphotoxin A (Muslta; GenBank: XM_006536550.2) as an unrelated gene absent in T. domestica. PCR amplification was performed using TaKaRa Ex Taq. The amplified fragments were retrieved from a 1.2\% agarose gel using an AxyPrep DNA Gel Extraction Kit (Axygen Scientific, Union City, CA, USA) and were then cloned into the pGEM-T Easy Vector (Promega, Madison, WI, USA) following the manufacturer's protocol. All positive clones were finally validated by DNA sequencing.

Prepared plasmids served as templates for dsRNA synthesis. In vitro transcriptional synthesis and purification of dsRNA were performed using a T7 RiboMAX ${ }^{\mathrm{TM}}$ Express RNAi System (Promega, Madison, WI, USA). The final concentration was adjusted to 6 $\mu \mathrm{g} / \mu \mathrm{L}$ with a NanoDrop2000, and all dsRNAs were stored at $-80^{\circ} \mathrm{C}$ until use. RNAi treatments were performed by body microinjection of dsRNA into N8 nymphs. Firebrats were anesthetized with carbon dioxide, and $200 \mathrm{~nL}$ of purified dsRNA was injected into the interlaminar fold of the abdomen per insect at a slow speed using a Nanoinject II microinjector apparatus (Drummond Scientific, Broomall, PA, USA). Glass capillaries (3.5 inc 3-000-203-G/X micropipettes, Drummond Scientific, Broomall, PA, USA) were prepared by a P-97 Micropipette Puller (Sutter Instrument Co., Novato, CA, USA) under the following parameters: heat $\frac{1}{4} 428$, pull $\frac{1}{4} 200$ and VEL $\frac{1}{4} 10$. The specimens were collected $72 \mathrm{~h}$ after the treatment and subjected to detection of RNAi efficiency using the whole body. 


\subsection{Starvation Treatment}

One-day-old N8 nymphs were starved or normally fed for $72 \mathrm{~h}$. To avoid cannibalism, insects were kept individually. The whole bodies of specimens were collected and subjected to RNA extraction, cDNA synthesis, and qPCR analysis. Following dissection, the tissues were stored at $-80^{\circ} \mathrm{C}$ until RNA was isolated. Three insects were pooled to constitute one biological replicate, and three biological replicates were analyzed for fed and starved T. domestica.

\subsection{Statistical Analysis}

The average $C_{t}$ value was calculated based on three biological replicates. The stability of the five candidate reference genes was evaluated using a web-based comprehensive tool (https:/ / www.heartcure.com.au/reffinder/?type=reference), which contained five universal analysis programs: comparative $\triangle C_{t}$ method [10], NormFinder [11], geNorm [12], BestKeeper [13], and RefFinder [14]. GeNorm calculated the ' $M$ ' value, with a lower value indicating a more stable expression. The NormFinder algorithm utilizes a linear mixed effect model to estimate both intra- and intergroup variations and to rank the candidate reference genes by stability values (SV). BestKeeper uses $\mathrm{Ct}$ values of genes to infer their stability, taking into consideration the standard deviation (SD), $p$-value, and correlation coefficient of each gene. Consequently, a lower ' $\mathrm{CV}$ ' value signifies the suitability of a particular gene to serve as a better internal control. As a comprehensive tool, RefFinder integrates the results from geNorm, NormFinder, BestKeeper, and the comparative Ct method and then ranks candidate reference genes based on the geometric mean values (GM).

To determine the optimal number of reference genes for normalization, the pairwise variations $V_{n / n+1}$ ( $n$ is the number of reference genes) were calculated between two sequential normalization factors $\left(\mathrm{NF}_{\mathrm{n}}\right.$ and $\left.\mathrm{NF}_{\mathrm{n}+1}\right)$ by geNorm with a cut-off value of 0.15 [12].

\section{Results}

\subsection{Expression Profiles of Candidate Reference Genes}

Under specific conditions at different developmental stages and in different body parts of $T$. domestica, all calculated $\mathrm{Ct}$ values of the five candidate genes varied from 17.00 to 27.01. According to these $\mathrm{Ct}$ values, EF1A showed the highest expression level, while SOD2 showed the lowest (Figure 1). Based on the standard deviation (SD) values, RPL32 showed the lowest expression variations among different samples (mean Ct value $\pm \mathrm{SD}=20.44 \pm 0.64$ ), and Actin5C showed the highest expression variations (mean Ct value $\pm \mathrm{SD}=22.59 \pm 1.31$ ) among the five genes. Rankings are shown in Table 1 when the results from all the tested samples were summarized and analyzed by the $\triangle C_{t}$ method, NormFinder, geNorm, BestKeeper, and RefFinder.

Table 1. Expression stability ranking of the selected candidate reference genes using Delta Ct, geNorm, BestKeeper, NormFinder, and RefFinder.

\begin{tabular}{|c|c|c|c|c|c|c|c|c|c|c|c|}
\hline \multirow[b]{2}{*}{ Rank } & \multicolumn{3}{|c|}{ Delta Ct } & \multicolumn{2}{|c|}{ geNorm } & \multicolumn{2}{|c|}{ BestKeeper } & \multicolumn{2}{|c|}{ NormFinder } & \multicolumn{2}{|c|}{ RefFinder } \\
\hline & $\begin{array}{l}\text { Gene } \\
\text { Name }\end{array}$ & $\begin{array}{c}\text { Average } \\
\text { Ct }\end{array}$ & SD & $\begin{array}{l}\text { Gene } \\
\text { Name }\end{array}$ & $\mathbf{M}$ & $\begin{array}{l}\text { Gene } \\
\text { Name }\end{array}$ & $\mathrm{CV}$ & $\begin{array}{l}\text { Gene } \\
\text { Name }\end{array}$ & SV & $\begin{array}{l}\text { Gene } \\
\text { Name }\end{array}$ & GM \\
\hline 1 & RPS26 & 21.08 & 0.75 & RPS26 & 0.39 & RPL32 & 2.48 & RPS26 & 0.27 & RPS26 & 1.19 \\
\hline 2 & RPL32 & 20.44 & 0.83 & RPL32 & 0.39 & RPS26 & 2.49 & RPL32 & 0.46 & RPL32 & 1.41 \\
\hline 3 & $E F 1 A$ & 18.61 & 0.91 & $E F 1 A$ & 0.62 & SOD2 & 3.39 & $E F 1 A$ & 0.63 & $E F 1 A$ & 3.22 \\
\hline 4 & SOD2 & 23.68 & 0.96 & SOD2 & 0.74 & EF1A & 4.48 & SOD2 & 0.69 & SOD2 & 3.72 \\
\hline 5 & Actin5C & 22.58 & 1.24 & Actin5C & 0.94 & Actin5C & 4.68 & Actin5C & 1.12 & Actin5C & 5.00 \\
\hline
\end{tabular}

Note: SD, standard deviations; $\mathrm{M}$, global gene expression stability values; $\mathrm{CV}$ : coefficient of variation; $\mathrm{SV}$, stability values; $\mathrm{GM}$, geometric mean. 


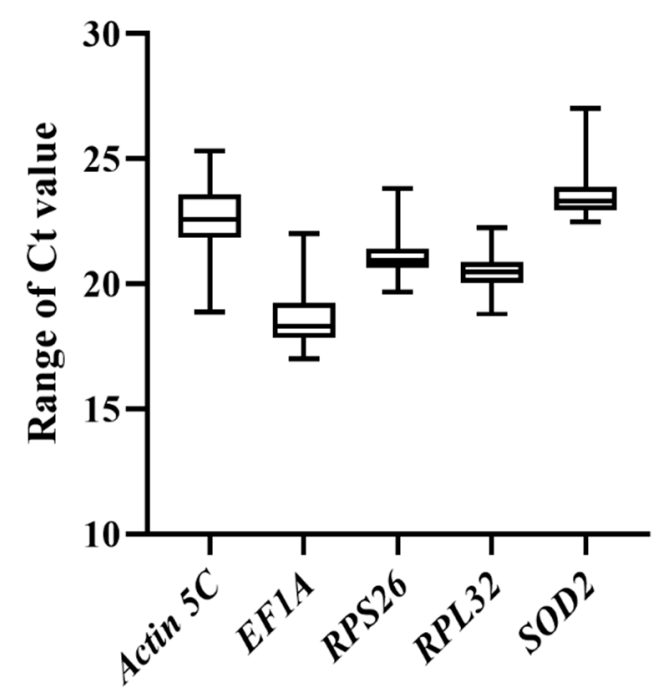

Figure 1. Ct value distribution of five reference genes in all analyzed samples of Thermobia domestica. The whiskers of the boxes are the minimum and maximum $\mathrm{Ct}$ values, and the horizontal lines inside the boxes are the median values.

\subsection{Evaluation of Candidate Genes for Gene Expression in Different Body Parts at Specific Developmental Stages of T. domestica}

The expression levels of the five candidate genes in five body parts $(\mathrm{H}, \mathrm{T} 1, \mathrm{~T} 23, \mathrm{~A}$, and $\mathrm{WB})$ at selected developmental stages (N6-N9, A1, A3, and A4) were determined by qPCR and analyzed for their expression stabilities by the four statistical algorithms. At developmental stages N6, N7, and A4 of T. domestica, geNorm, BestKeeper, and NormFinder analyses obtained consistent results showing that RPS26 and RPL32 were the most stably expressed genes in different body parts (Figure 2A,B,G). At developmental stages N8, N9, $\mathrm{A} 1$, and A3 of T. domestica, the gene expression of RPS26 and RPL32 was also most stable based on the geNorm and NormFinder algorithms, but BestKeeper analysis showed that SOD2 and RPL32 were the two best choices (Figure 2C-F). Summarized rankings from RefFinder analysis showed that RPS26 and RPL32 were the most stably expressed genes in different body parts at all tested developmental stages of $T$. domestica, while the expression of Actin5C was the least stable in different body parts at all tested developmental stages except at N6, in which EF1A was more altered than Actin5C (Figure 2).

\subsection{Evaluation of Candidate Genes for Gene Expression at Different Developmental Stages in Specific Body Parts of T. domestica}

To determine the expression of these candidate genes at different developmental stages in some specific $T$. domestica body parts, we analyzed the expression stability of five candidate genes through qPCR for the developmental stages N6-N9, A1, A3, and A4 in the head, prothorax, mesothorax/metathorax, abdomen, and whole body by using the four algorithms mentioned above. In the head, SOD2 and RPS26 were most stable at different developmental stages based on all four analyses (Figure 3A). In the prothorax, SOD2 and RPS26 were also optimal according to the geNorm and BestKeeper algorithms, while SOD2 and RPL32 were preferred by NormFinder analysis (Figure 3B). In the mesotho$\mathrm{rax} /$ metathorax and whole body, SOD2 and RPS26 were recommended by geNorm and BestKeeper analyses, while RPL32 and RPS26 were favored by the NormFinder algorithm (Figure 3C,E); in the abdomen of T. domestica, RPL32 and RPS26 were selected by all algorithms (Figure 3D). According to the comprehensive ranking analysis of RefFinder, SOD2 and RPS26 were the two most stably expressed genes at different developmental stages in the head, prothorax, mesothorax/metathorax, and whole body of $T$. domestica, respectively, while RPL32 and RPS26 were the most appropriate reference genes for gene expression studies in the abdomen of $T$. domestica. In addition, the least stable genes at different 
developmental stages were Actin $5 \mathrm{C}$ in the head, prothorax and mesothorax/metathorax, $S O D 2$ in the abdomen, and EF1A in the whole body.
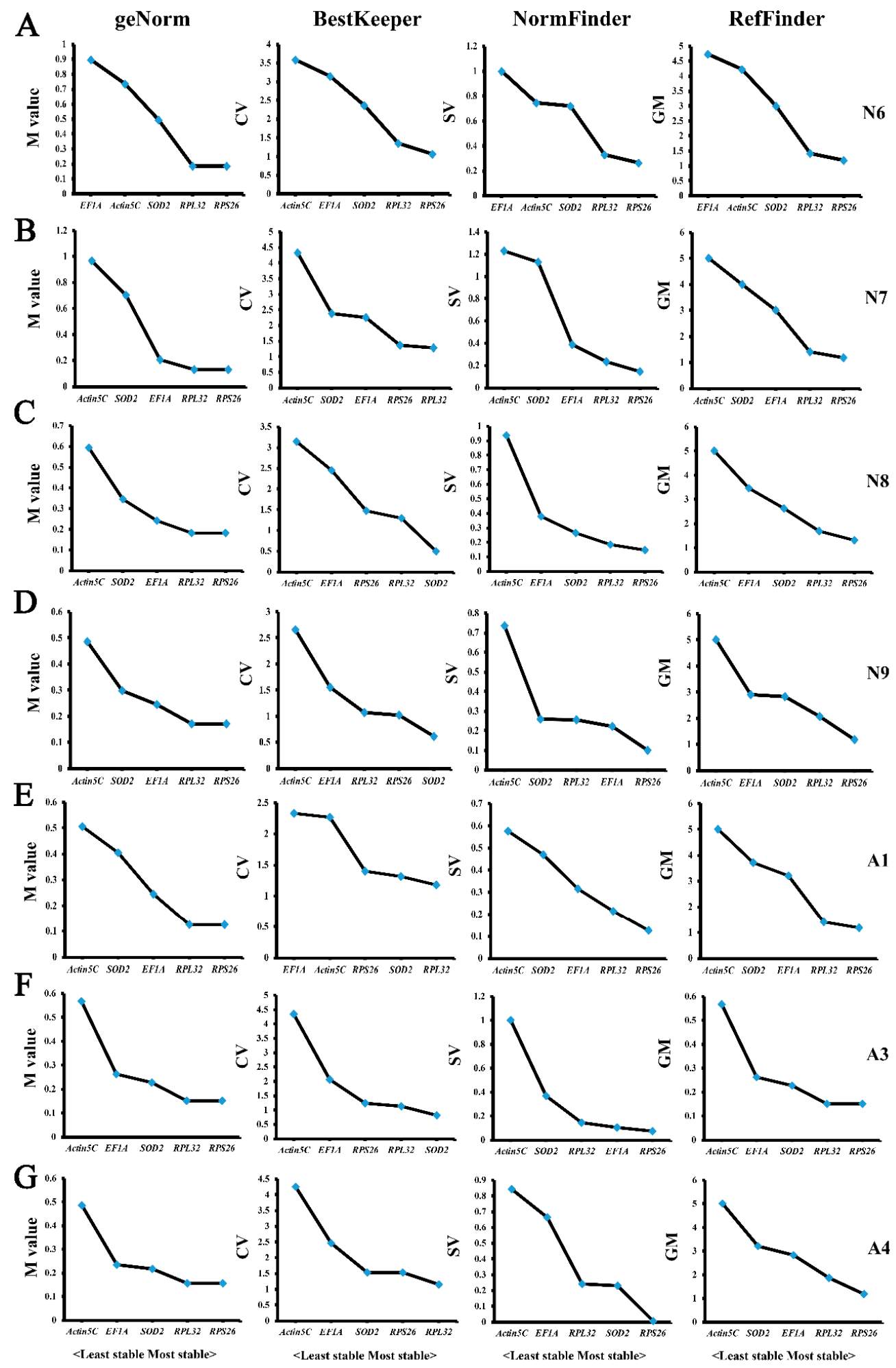

Figure 2. Stable analysis of five candidate genes in different tissues of Thermobia domestica (head, prothorax, mesothorax/metathorax, abdomen, and whole body) at the developmental stages of nymphs N6 (A), N7(B), N8 (C), and N9 (D), and adults A1 (E), A3 (F), and A4 (G) based on four algorithms. 
$\mathbf{A}$ geNorm
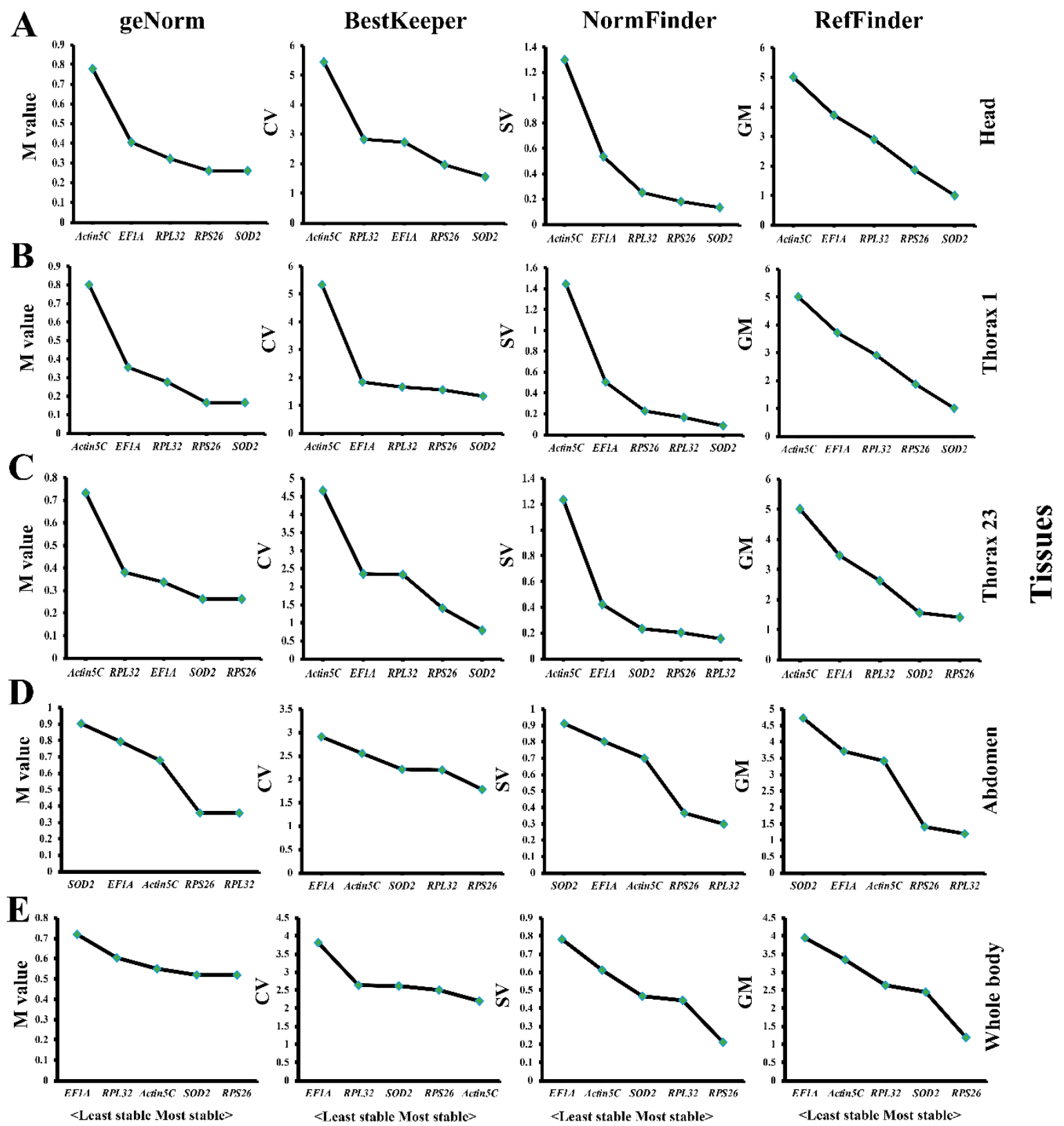

Figure 3. Stable analysis of five candidate genes at different developmental stages from nymphs (N6-N9) and adults (A1, A3, and A4) of Thermobia domestica in different tissues: head (A), prothorax (B), mesothorax/metathorax (C), abdomen (D), and whole body (E) based on four algorithms.

\subsection{Evaluation of Candidate Genes for Gene Expression in Tdmyo-Silenced and Starvation Conditions}

To determine whether RNAi affects the expression of the five candidate genes, we silenced Myo expression in T. domestica through microinjection and verified the Myo expression level by qPCR analysis. Muslta, a gene absent in T. domestica, was used as the control. Analysis of the gene expression of five candidate genes based on the qPCR data from the whole body samples injected with dsRNA of Tdmyo or Muslta showed that EF1A and $R P L 32$ were the most stably expressed genes using the geNorm, BestKeeper, NormFinder, and RefFinder algorithms (Figure 4A). 

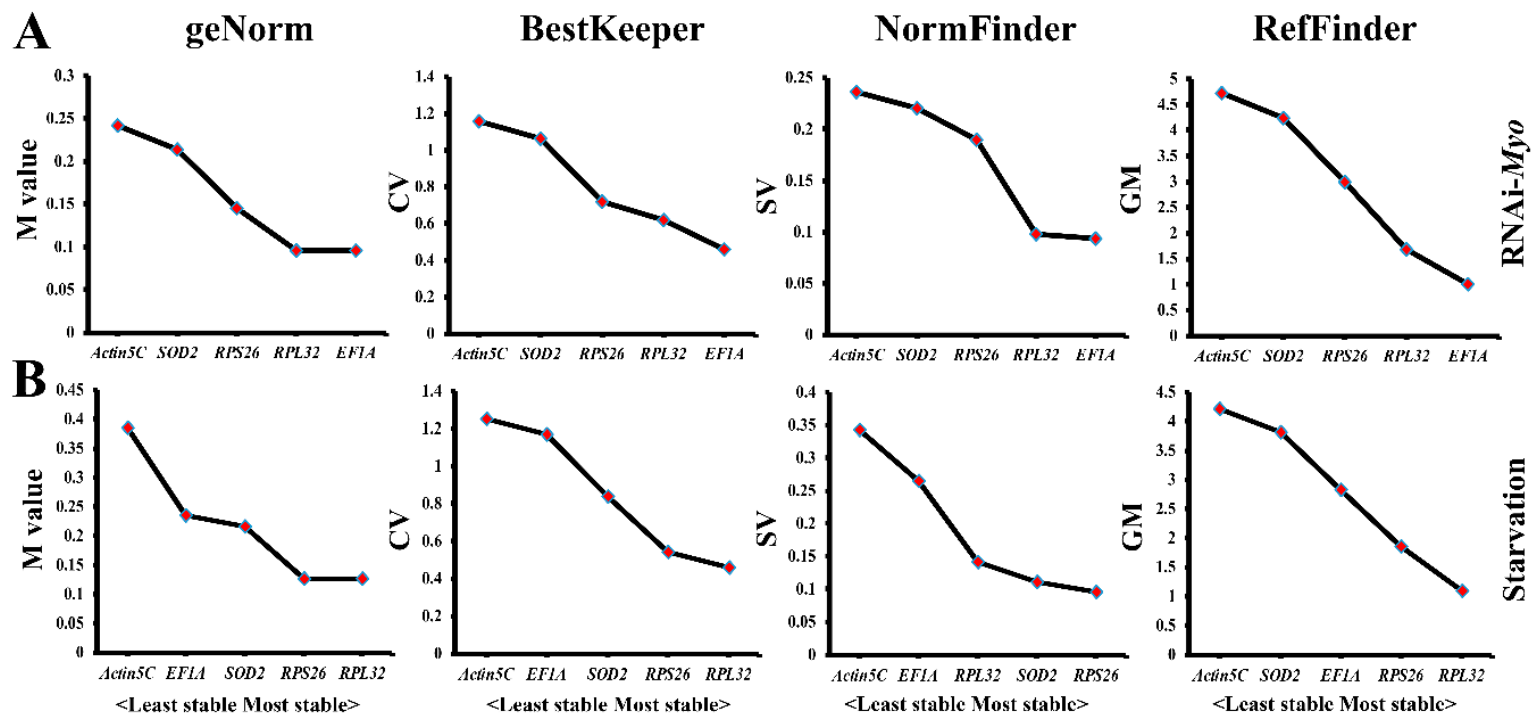

Figure 4. Stable analysis of five candidate genes under RNAi-Myo (A) or starvation stresses (B) based on four algorithms.

To further evaluate the effect of starvation stress, we compared the expression of five candidate genes in T. domestica whole body samples under fed or starved conditions through qPCR. Analyses with the geNorm and BestKeeper algorithms showed that RPL32 and RPS26 were the most stably expressed genes under starvation stress, but RPS26 and SOD2 were optimal using NormFinder analysis. The comprehensive stability ranking of the five candidate genes generated by the RefFinder algorithm was RPL32 > RPS26 > EF1A $>$ SOD2 > Actin5C (Figure 4B).

\subsection{Determination of the Optimal Number of Reference Genes Needed for qPCR Normalization}

To reduce the probability of biased normalization, multiple reference genes have been used to normalize target gene expression [15-17]. However, either too few or too many reference genes may be detrimental to the accuracy of target gene expression $[18,19]$. Based on the evaluated rankings of all experimental sets in Figures 2 and 3, the pairwise variation values between ranked genes $\left(V_{n / n+1}\right)$ were calculated by the geNorm algorithm to demonstrate the effect of adding reference genes on the stability of the normalization factor, and to determine the optimal number of reference genes in T. domestica using a cutoff of 0.15 [12]. A threshold value below 0.15 indicated that the additional reference gene had no significant improvement on normalization in qPCR data.

At each studied developmental stage of nymphs (N6-N9) and adults (A1, A3, and A4), the $V_{2 / 3}$ value for all experimental sets in different tissues was lower than 0.15 (Figure 5A), suggesting that two reference genes can standardize these samples well. According to the rankings in Figure 2, RPS26 and RPL32 together were the optimum reference gene combination for $\mathrm{qPCR}$ analysis in different body parts at the selected stages of middle and late nymphs (N6-N9) and adults (A1, A3, and A4). In contrast, Actin5C introduced negative effects at stages N7 and $A 4$, since their $V_{4 / 5}$ values increased obviously. 

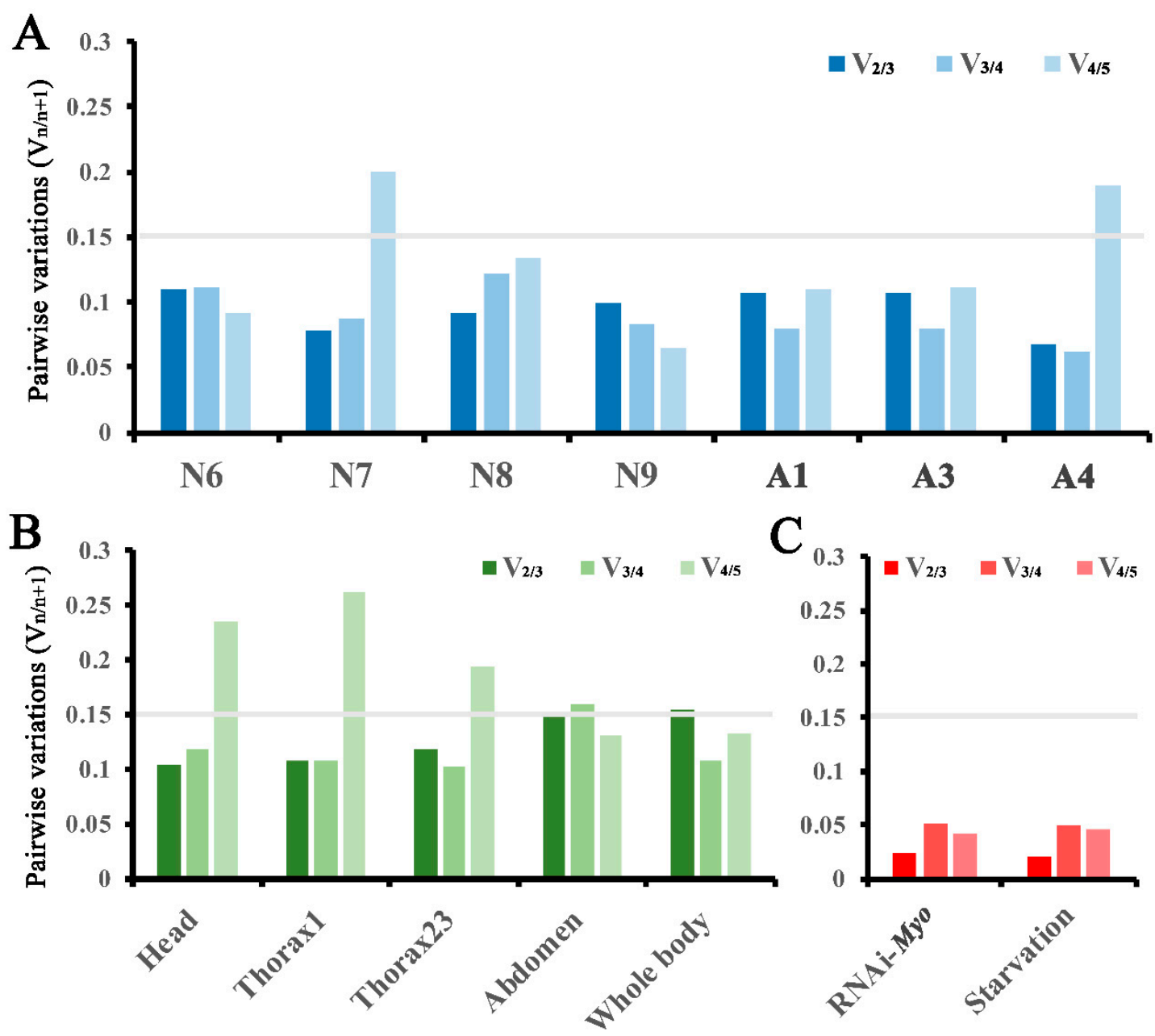

Figure 5. Evaluation of the optimal number of reference genes for normalization in Thermobia domestica. The pairwise variations $(\mathrm{V} n / n+1, n$ is the number of reference genes) was calculated between two sequential normalization factors $\left(\mathrm{NF}_{\mathrm{n}}\right.$ and $\left.\mathrm{NF}_{\mathrm{n}+1}\right)$ by geNorm with a cut-off value of 0.15 [12] using data: (A) from different tissues at the developmental stages of N6-N9, A1, A3, and A4; (B) at different developmental stages in the head, prothorax, mesothorax/metathorax, and abdomen; $(\mathbf{C})$ in whole insects at the 8 th nymphal instar under RNAi and starvation treatments.

In the five investigated body parts, the $\mathrm{V}_{2 / 3}$ values at different developmental stages were apparently lower than 0.15 in the head, prothorax, and mesothorax/metathorax of T. domestica and were very close to 0.15 in the abdomen and whole body (Figure 5B). Thus, two reference genes would be effective for standardizing these samples. Based on the evaluation ranking in Figure 3, the combination of SOD2 and RPS26 was the optimum for qPCR analysis at different stages in the head and thorax, the combination of RPL32 and RPS26 was ideal in the abdomen, and the combination of RPS26 and SOD2 was a suitable normalization in the whole body. Additionally, the inclusion of Actin5C, the least stable gene in the head, prothorax, and mesothorax/metathorax, greatly increased the corresponding $\mathrm{V}_{4 / 5}$ values in each tissue.

Under the Tdmyo-silenced and starvation conditions, all tested combinations were lower than 0.05 , and both $\mathrm{V}_{2 / 3}$ values were the lowest (Figure 5C). Based on the evaluation ranking in Figure 4, the combination of EF1A and RPL32 for RNAi-Tdmyo and the combination of RPL32 and RPS26 for starvation treatment were the best choices.

Overall, the combination of RPL32 and RPS26 was the best choice for most tested samples, except for samples at different stages in the head, thorax, and whole body (RPS26 and SOD2), and under RNAi-Tdmyo treatment (EF1A and RPL32). However, the inclusion of RPL32 at different stages in the head, thorax, and whole body had no significant effect 
on the corresponding pairwise variation (Figure 5B), neither did the inclusion of RPS26 under RNAi-Tdmyo treatment (Figure 5C). Using the same reference standard for all the studies would be best to be able to compare data. Thus, the combination of RPS26 and $R P L 32$ is recommended for all tested experimental conditions.

\subsection{Normalization of qPCR Data of Tdmyo Using Selected Reference Genes}

Myo has been proven to trigger the premetamorphosis stage in hemimetabolan insects [7], and it is worth studying Myo expression in ametabolous insects to understand its genetic evolution. Normalized with RPS26 and RPL32, the relative expression level of Tdmyo in the head of T. domestica was similar at N6 and N7, increased remarkably and reached the highest level in N8 (the penultimate nymphal instar), was slightly lower but remained high in N9 (the last nymphal instar), and then progressively decreased in adults (Figure 6A). At N8 of T. domestica, the head showed the highest Myo mRNA levels, lower Myo expression was indicated in the abdomen and mesothorax/metathorax, and the lowest expression was observed in the prothorax (Figure 6B).
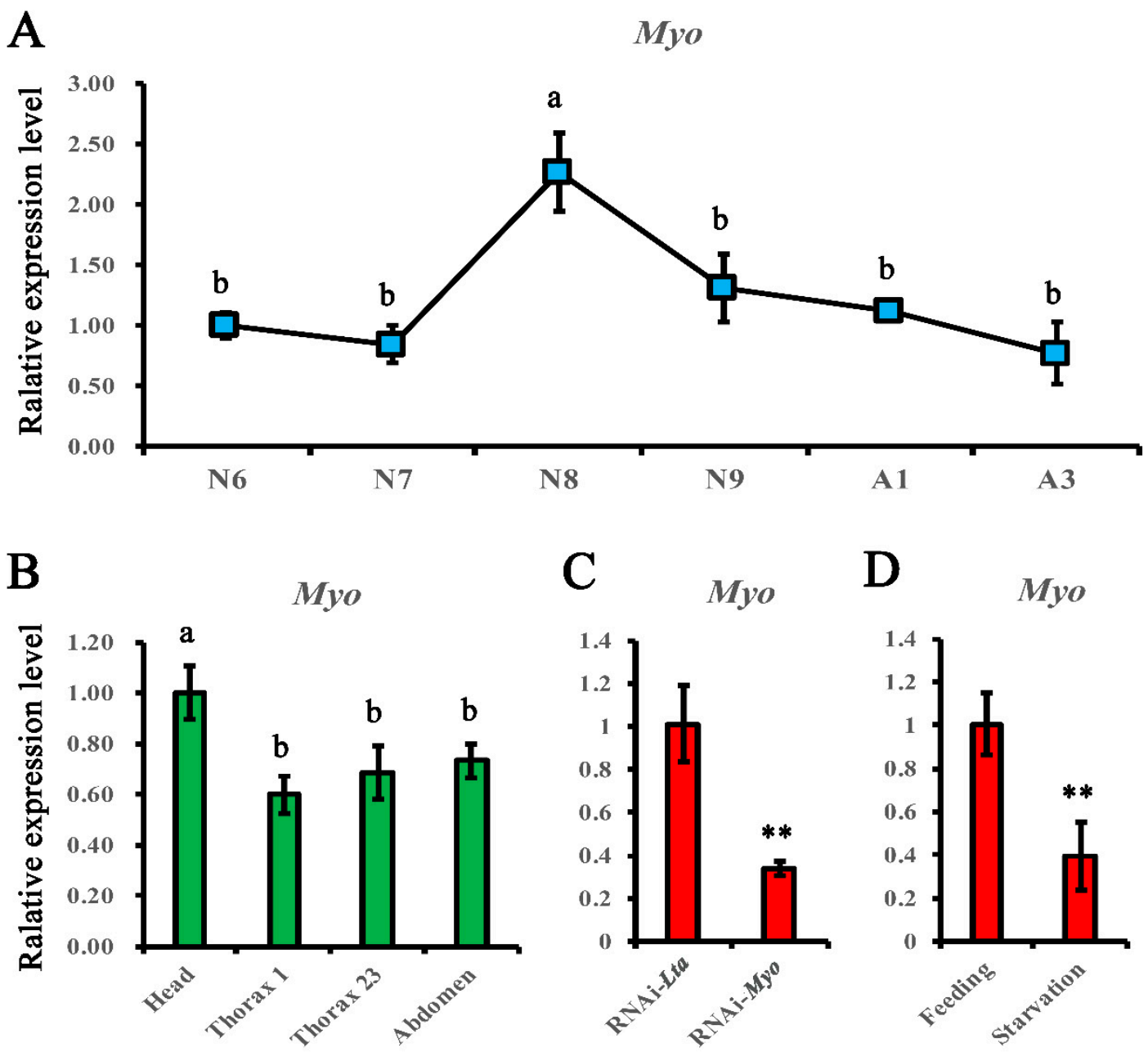

Figure 6. Relative expression levels of Tdmyo under various experimental conditions normalized with RPS26 and RPL32. Error bars show the mean standard error calculated from three biological replicates. (A) In the head of T. domestica from the middle nymphs to adults. (B) In four body parts from individuals at the 8th nymphal instar. (C) In whole insects at the 8th nymphal instar under RNAi treatment. (D) In whole insects at the 8th nymphal instar under feeding or starvation conditions. Different letters $(a, b)$ in $(\mathbf{A}, \mathbf{B})$ indicate significant differences of values (ANOVA, LSD, $p<0.05)$. The statistical level in $(\mathbf{C}, \mathbf{D})$ was assessed with ${ }^{* *} p<0.01$. 
Under RNAi conditions at the 8th instar of T. domestica, the Tdmyo mRNA level was significantly reduced in $d s T d m y o$-treated firebrats compared with dsMuslta-treated firebrats (Figure 6C), which suggested a high efficiency of RNAi Myo in firebrat nymphs.

Similarly, Myo mRNA levels were significantly downregulated in the starvation treatment group compared with the fed groups (Figure 6D), demonstrating that nutrition can notably affect Myo expression.

\section{Discussion}

qPCR is an efficient and convenient method for gene expression analysis, and using an appropriate reference gene or genes is the basic requirement to achieve qPCR reliability. To date, the selection of reliable reference genes under different conditions has been performed in many insect and mite species [8] such as locusts [20,21], pea aphids [22], psocids [23], honey bees [24], wasps [25], cotton bollworms [26], flies [27,28], silkworms [29], moths [30], beetles [31-33] and spider mites [34-36], but not the firebrat T. domestica, a representative basal insect, which plays a key role in understanding the origin of insect metamorphosis. To develop functional genomic studies in this insect, it is essential to select optimal reference genes and test the efficacy of RNAi. In this study, we comprehensively evaluated the expression of five candidate genes (Actin5C, EF1A, RPS26, RPL32, and SOD2) in T. domestica under various conditions, including in different tissues at some specific developmental stages, at different developmental stages in several specific tissues, and under dsRNA microinjection and starvation stresses. Most rankings obtained from different algorithms (BestKeeper, geNorm, NormFinder, and RefFinder) were consistent, which demonstrated the reliability of our results.

Actin, a traditional housekeeping gene, has been regarded as a stable reference gene across insect species in various experimental settings [20,21,24,37] but not in beetles [31-33]. Elongation factor 1 alpha $(E F-1 \alpha)$, an evolutionarily conserved GTPase, was evaluated as the stable gene in Orthoptera [21], Drosophila [37], and Hymenoptera [38]. However, in this work, both Actin5C and EF-1 $\alpha$ were ranked as the two least stable reference genes under most experimental conditions (Figures 2 and 3). In particular, the pairwise variation values were increased greatly by introducing Actin5C (Figure 5) in different tissues at the 7 th nymphal stage and the 4 th adult stage, as well as at different developmental stages in the head, prothorax, and mesothorax/metathorax (Figure 4). Our analyses proposed that Actin 5C and EF-1 $\alpha$ were not appropriate to reference genes in T. domestica.

Various ribosomal protein genes have been evaluated and showed highly stable expression in insects [8] and mites, such as RPS9 and RPL13 in the spider mite [35,36], RPS18 in honey bee [24], the spider mite [34], bloodsucking bug [39], RPL18 in the bed bug [40], and RP49/RPL32 in the desert locust [20]. Our results suggested that the RPS26 and RPL32 genes were the two most stable genes among the five candidates in different tissues at all developmental stages tested (nymphs and adults) in T. domestica (Figure 2). In addition, both RPS26 and RPL32 were more stable than Actin5C and EF-1 $\alpha$ at different developmental stages in all studied tissues. SOD2 performed well at different developmental stages in the head, prothorax, mesothorax/metathorax, and whole body of T. domestica but worst in the abdomen (Figure 3). Our results further prove the importance of selecting stable reference genes before each qPCR under specific experimental conditions. Additionally, our pairwise variation analyses suggested that two reference genes, RPS26 and RPL32, can standardize all tested samples of T. domestica well.

The postembryonic development period is essential for insect growth, accumulation of reserves, and metamorphosis. To date, there are few RNAi studies on nymph stages in Zygentoma. This study demonstrated a high RNAi efficiency of Myo in firebrat nymphs. The Tdmyo mRNA level was significantly reduced in dsTdmyo-treated nymphs at the 8th instar (Figure 6C). Myo is a key trigger of the transition from the penultimate to final (premetamorphic) nymphal stage in the hemimetabolan cockroach [7]. With normalization to the selected internal control genes, RPS26 and RPL32, we performed qPCR to study Myo expression patterns in firebrat heads at different developmental stages and in different 
tissues at the 8th nymphal instar, as well as Myo expression changes under RNAi-Myo and starvation treatments. In the head of $T$. domestica, the highest expression was observed in N8 (Figure 6A), the penultimate nymphal instar, in accordance with the expression pattern in the cockroach [7], indicating its conserved function in hemimetabolous cockroach and ametabolous firebrat.

\section{Conclusions}

In summary, we evaluated five candidate reference genes in T. domestica, a representative ametabolous insect, under various experimental conditions. Similar rankings of five candidate genes were obtained by different algorithms, and the optimal number of reference genes was calculated as two for most experimental sets. The top two stable reference genes were as follows: (1) RPS26 and RPL32 in different body parts at all tested developmental stages, (2) SOD2 and RPS26 at different developmental stages in the head, prothorax, mesothorax/metathorax, and whole body, (3) RPS26 and RPL32 at different developmental stages in the abdomen, (4) RPL32 and EF1A under microinjection of dsRNA (Myoglianin, Myo), and (5) RPS26 and RPL32 under starvation stress. Overall, the combination of RPS26 and RPL32 was recommended as a suitable reference for all settings performed in this study. In contrast, the expression of Actin5C was least stable in most tested samples. By exploring qPCR and RNA $i$ in T. domestica, we found that Myo in wingless $T$. domestica had a similar spatial and temporal expression patterns with those of winged insects, and RNAi effect is prominent in the nymphal stage. Our findings not only help to establish a more accurate and reliable normalization of real-time qPCR data in T. domestica but also lay a solid foundation for further studies of RNA interference and gene transcription in basal apterous insects.

Supplementary Materials: The following are available online at https:/ www.mdpi.com/2073-442 $5 / 12 / 1 / 21 / s 1$, Figure S1: Melting curves, melting peaks and amplification curves of the five selected candidate reference genes. Table S1: Primers of the candidate reference genes used in this study. Table S2: Primers used for qRT-PCR analysis of Myo gene expression and dsRNA synthesis.

Author Contributions: Conceptualization: Y.B., Y.-Y.C., and Y.-X.L.; methodology: Y.B., Y.-N.L., M.Z., P.-Y.J., H.-N.L., and Y.-B.Z.; data analysis: Y.B., Y.-N.L., M.Z., P.-Y.J., H.-N.L., and Y.-B.Z.; writing-original draft preparation, Y.B., Y.-Y.C., and Y.-X.L.; writing-review and editing, S.L.; funding acquisition: Y.-Y.C. and Y.-X.L. All authors have read and agreed to the published version of the manuscript.

Funding: This work was supported by the National Natural Science Foundation of China (31970438, 31772510, 31900342) and the Natural Science Foundation of Guangdong Province (2018A030313130).

Acknowledgments: The authors thank Peng Gao (Beijing Forestry University, China) for his assistance in data processing.

Conflicts of Interest: The authors declare no conflict of interest.

\section{References}

1. Misof, B.; Liu, S.; Meusemann, K.; Peters, R.S.; Donath, A.; Mayer, C.; Frandsen, P.B.; Ware, J.; Flouri, T.; Beutel, R.G.; et al. Phylogenomics resolves the timing and pattern of insect evolution. Science 2014, 346, 763-767. [CrossRef]

2. Brand, P.; Robertson, H.M.; Lin, W.; Pothula, R.; Klingeman, W.E.; Jurat-Fuentes, J.L.; Johnson, B.R. The origin of the odorant receptor gene family in insects. eLife 2018, 7, 38340. [CrossRef] [PubMed]

3. Kamae, Y.; Tomioka, K. Timeless is an essential component of the circadian clock in a primitive insect, the firebrat Thermobia domestica. J. Biol. Rhythm. 2012, 27, 126-134. [CrossRef] [PubMed]

4. Kamae, Y.; Uryu, O.; Miki, T.; Tomioka, K. The nuclear receptor genes HR3 and E75 are required for the circadian rhythm in a primitive insect. PLOS ONE 2014, 9, e114899. [CrossRef] [PubMed]

5. Ohde, T.; Masumoto, M.; Yaginuma, T.; Niimi, T. Embryonic RNAi analysis in the firebrat, Thermobia domestica: Distal-less is required to form caudal filament. J. Insect Biotech. Seric. 2009, 78, 99-105. [CrossRef]

6. Ishimaru, Y.; Tomonari, S.; Matsuoka, Y.; Watanabe, T.; Miyawaki, K.; Bando, T.; Tomioka, K.; Ohuchi, H.; Noji, S.; Mito, T. TGF- $\beta$ signaling in insects regulates metamorphosis via juvenile hormone biosynthesis. Proc. Natl. Acad. Sci. USA 2016, 113, 5634-5639. [CrossRef] 
7. Kamsoi, O.; Belles, X. Myoglianin triggers the premetamorphosis stage in hemimetabolan insects. FASEB J. 2018, 33, 3659-3669. [CrossRef]

8. Lü, J.; Yang, C.; Zhang, Y.; Pan, H. Selection of reference genes for the normalization of RT-qPCR data in gene expression studies in insects: A systematic review. Front Physiol. 2018, 9, 1560. [CrossRef]

9. Bustin, S.A.; Benes, V.; Garson, J.A.; Hellemans, J.; Huggett, J.; Kubista, M.; Mueller, R.; Nolan, T.; Pfaffl, M.W.; Shipley, G.L.; et al. The MIQE guidelines: Minimum information for publication of quantitative real-time PCR experiments. Clin. Chem. 2009, 55, 611-622. [CrossRef]

10. Silver, N.; Best, S.; Jiang, J.; Thein, S.L. Selection of housekeeping genes for gene expression studies in human reticulocytes using real-time PCR. BMC Mol. Biol. 2006, 7, 33. [CrossRef]

11. Andersen, C.L.; Jensen, J.L.; Orntoft, T.F. Normalization of real-time quantitative reverse transcription-PCR data: A model-based variance estimation approach to identify genes suited for normalization, applied to bladder and colon cancer data sets. Cancer Res. 2004, 64, 5245-5250. [CrossRef] [PubMed]

12. Vandesompele, J.; De Preter, K.; Pattyn, F.; Poppe, B.; Van Roy, N.; De Paepe, A.; Speleman, F. Accurate normalization of real-time quantitative RT-PCR data by geometric averaging of multiple internal control genes. Genome Biol. 2002, 3, research0034.1. [CrossRef] [PubMed]

13. Pfaffl, M.W.; Tichopad, A.; Prgomet, C.; Neuvians, T.P. Determination of stable housekeeping genes, differentially regulated target genes and sample integrity: BestKeeper-Excel-based tool using pair-wise correlations. Biotechnol. Lett. 2004, 26, 509-515. [CrossRef] [PubMed]

14. Xie, F.; Xiao, P.; Chen, D.; Xu, L.; Zhang, B. miRDeepFinder: A miRNA analysis tool for deep sequencing of plant small RNAs. Plant. Mol. Biol. 2012, 80, 75-84. [CrossRef]

15. Tatsumi, K.; Ohashi, K.; Taminishi, S.; Okano, T.; Yoshioka, A.; Shima, M. Reference gene selection for real-time RT-PCR in regenerating mouse livers. Biochem. Biophys. Res. Commun. 2008, 374, 106-110. [CrossRef] [PubMed]

16. Shakesby, A.; Wallace, I.; Isaacs, H.; Pritchard, J.; Roberts, D.; Douglas, A. A water-specific aquaporin involved in aphid osmoregulation. Insect Biochem. Mol. Biol. 2009, 39, 1-10. [CrossRef]

17. Pitino, M.; Coleman, A.D.; Maffei, M.E.; Ridout, C.J.; Hogenhout, S.A. Silencing of aphid genes by dsRNA feeding from plants. PLoS ONE 2011, 6, e25709. [CrossRef]

18. Ling, D.; Salvaterra, P.M. Robust RT-qPCR data normalization: Validation and selection of internal reference genes during post-experimental data analysis. PLOS ONE 2011, 6, e17762. [CrossRef]

19. Fu, W.; Xie, W.; Zhang, Z.; Wang, S.; Wu, Q.; Liu, Y.; Zhou, X.; Zhou, X.; Zhang, Y. Exploring valid reference genes for quan-titative real-time PCR analysis in Plutella xylostella (Lepidoptera: Plutellidae). Int. J. Biol. Sci. 2013, 9, 792-802. [CrossRef]

20. Van Hiel, B.; Van Wielendaele, P.; Temmerman, L.; Van Soest, S.; Vuerinckx, K.; Huybrechts, R.; Broeck, J.V.; Simonet, G. Identification and validation of housekeeping genes in brains of the desert locust Schistocerca gregaria under different developmental conditions. BMC Mol. Biol. 2009, 10, 56. [CrossRef]

21. Chapuis, M.; Tohidi-Esfahani, D.; Dodgson, T.; Blondin, L.; Ponton, F.; Cullen, D.A.; Simpson, S.J.; Sword, G.A. Assessment and validation of a suite of reverse transcription-quantitative PCR reference genes for analyses of density-dependent behavioural plasticity in the Australian plague locust. BMC Mol. Biol. 2011, 12, 7. [CrossRef] [PubMed]

22. Yang, C.; Pan, H.; Liu, Y.; Zhou, X. Selection of reference genes for expression analysis using quantitative real-time PCR in the pea aphid, Acyrthosiphon pisum (Harris) (Hemiptera, Aphidiae). PLoS ONE 2014, 9, e110454. [CrossRef] [PubMed]

23. Jiang, H.-B.; Liu, Y.-H.; Tang, P.-A.; Zhou, A.-W.; Wang, J. Validation of endogenous reference genes for insecticide-induced and developmental expression profiling of Liposcelis bostsrychophila (Psocoptera: Liposcelididae). Mol. Biol. Rep. 2009, 37, 1019-1029. [CrossRef] [PubMed]

24. Scharlaken, B.; de Graaf, D.C.; Goossens, K.; Brunain, M.; Peelman, L.J.; Jacobs, F.J. Reference gene election for insect expression studies using quantitative real-time PCR: The head of the honeybee, Apis mellifera, after a bacterial challenge. J. Insect Sci. 2008, 8, 33. [CrossRef]

25. Li, Q.-Y.; Li, Z.-L.; Lu, M.-X.; Cao, S.-S.; Du, Y.-Z. Selection of valid reference genes for quantitative real-time PCR in Cotesia chilonis (Hymenoptera: Braconidae) exposed to different temperatures. PLoS ONE 2019, 14, e0226139. [CrossRef]

26. Zhang, S.; An, S.; Li, Z.; Wu, F.; Yang, Q.; Liu, Y.; Gao, J.; Zhang, H.; Zhang, Q.; Liu, X. Identification and validation of reference genes for normalization of gene expression analysis using qRT-PCR in Helicoverpa armigera (Lepidoptera: Noctuidae). Gene 2015, 555, 393-402. [CrossRef]

27. Shen, G.-M.; Jiang, H.-B.; Wang, X.-N.; Wang, J. Evaluation of endogenous references for gene expression profiling in different tissues of the oriental fruit fly Bactrocera dorsalis (Diptera: Tephritidae). BMC Mol. Biol. 2010, 11, 76. [CrossRef]

28. Gao, Z.; Deng, W.; Zhu, F. Reference gene selection for quantitative gene expression analysis in black soldier fly (Hermetia illucens). PLoS ONE 2019, 14, e0221420. [CrossRef]

29. Peng, R.; Zhai, Y.; Ding, H.; Di, T.; Zhang, T.; Li, B.; Shen, W.; Wei, Z. Analysis of reference gene expression for real-time PCR based on relative quantitation and dual spike-in strategy in the silkworm Bombyx mori. Acta Biochim. Biophys. Sin. 2012, 44, 614-622. [CrossRef]

30. Liu, G.; Qiu, X.; Cao, L.; Zhang, Y.; Zhan, Z.; Han, R. Evaluation of reference genes for reverse transcription quantitative PCR studies of physiological responses in the ghost moth, Thitarodes armoricanus (Lepidoptera, Hepialidae). PLoS ONE 2016, 11, e0159060. [CrossRef] 
31. Yang, C.; Preisser, E.L.; Zhang, H.; Liu, Y.; Dai, L.; Pan, H.; Zhou, X. Selection of reference genes for RT-qPCR analysis in Coccinella septempunctata to assess un-intended effects of RNAi transgenic plants. Front. Plant Sci. 2016, 7, 1672. [CrossRef] [PubMed]

32. Gao, P.; Wang, J.; Wen, J.-B. Selection of reference genes for tissue/organ samples of adults of Eucryptorrhynchus scrobiculatus. PLoS ONE 2020, 15, e0228308. [CrossRef] [PubMed]

33. Zheng, C.; Zhao, D.; Xu, Y.; Shi, F.; Zong, S.; Tao, J. Reference gene selection for expression analyses by qRT-PCR in Dendroctonus valens. Insects 2020, 11, 328. [CrossRef] [PubMed]

34. Sun, W.; Jin, Y.; He, L.; Lu, W.-C.; Li, M. Suitable reference gene selection for different strains and developmental stages of the carmine spider mite, Tetranychus cinnabarinus, using quantitative real-time PCR. J. Insect Sci. 2010, 10, 208. [CrossRef]

35. Yang, J.; Gao, Y.; Liu, Z.; Lu, J.; Zhang, Y.; Zhang, P.; Fan, J.; Zhou, X.; Fan, R. Selection of reference genes for RT-qPCR analysis under intrinsic conditions in the hawthorn spider mite, Amphitetranychus viennensis (Acarina: Tetranychidae). Front. Physiol. 2019, 10, 1427. [CrossRef]

36. Yang, J.; Zhang, Y.; Zhao, J.; Gao, Y.; Liu, Z.; Zhang, P.; Fan, J.; Zhou, X.; Fan, R. Selection of reference genes for RT-qPCR analysis under extrinsic conditions in the hawthorn spider mite, Amphitetranychus viennensis. Front. Physiol. 2020, 11, 378. [CrossRef]

37. Ponton, F.; Chapuis, M.-P.; Pernice, M.; Sword, G.A.; Simpson, S.J. Evaluation of potential reference genes for reverse transcription-qPCR studies of physiological responses in Drosophila melanogaster. J. Insect Physiol. 2011, 57, 840-850. [CrossRef]

38. Horňáková, D.; Matoušková, P.; Kindl, J.; Valterová, I.; Pichová, I. Selection of reference genes for real-time polymerase chain reaction analysis in tissues from Bombus terrestris and Bombus lucorum of different ages. Anal. Biochem. 2010, 397, 118-120. [CrossRef]

39. Majerowicz, D.; Alves-Bezerra, M.; Logullo, R.; Fonseca-de-Souza, A.L.; Meyer-Fernandes, J.R.; Braz, G.R.C.; Gondim, K.C. Looking for reference genes for real-time quantitative PCR experiments in Rhodnius rolixus (Hemiptera: Reduviidae). Insect Mol. Biol. 2011, 20, 713-722. [CrossRef]

40. Mamidala, P.; Rajarapu, S.P.; Jones, S.C.; Mittapalli, O. Identification and validation of reference genes for quantitative re-al-time polymerse chain reaction in Cimex lectularius. J. Med. Entomol. 2011, 48, 947-951. [CrossRef] 